\title{
A Study of 4D Printing and Functionally Graded Additive Manufacturing
}

\author{
Eujin Pei, Giselle Hsiang Loh and David Harrison \\ Department of Design, Brunel University, London, UK
}

\section{Henrique de Amorim Almeida}

Department of Mechanical Engineering, School of Technology and Management, Polytechnic Institute of Leiria, Leiria, Portugal, and

Mario Domingo Monzón Verona and Rubén Paz

Universidad de Las Palmas de Gran Canaria, Las Palmas, Spain

\section{Keywords}

Functionally Graded Additive Manufacturing, 4D Printing, Functionally Graded Rapid Prototyping, Varied Property Rapid Prototyping

\begin{abstract}
The purpose of this paper is to clarify the concept of Functionally Graded Additive Manufacturing and 4D printing. We defined that Functionally Graded Additive Manufacturing (FGAM) is a single AM process that includes the gradationally mixing of materials to fabricate freeform geometries with variable-properties within one component. This should not be used interchangeably with the term 4D Printing in which refers to the use of smart materials in Additive Manufacturing to produce parts that have the ability to change when exposed to an environmental stimuli. In this paper, we highlight that FGAM requires better computational tools for modelling, simulation and fabrication as current CAD systems are incapable of supporting the complex workflow, suggesting that future work should focus on aspects of material characterization and better control processes.
\end{abstract}

\subsection{Introduction to Functionally Graded Additive Manufacturing}

Additive Manufacturing (AM), commonly known as 3D Printing or Rapid Prototyping (RP) has the ability to produce parts with a freedom of geometry and enabling the operator to fully control the production process chain. Technological progress in hardware, software, as well as the opening of new markets and applications has encouraged research into novel processes and materials for AM to a large extent.

The purpose of this paper is to clarify the concept of Functionally Graded Additive Manufacturing and to distinguish it from 4D printing. Functionally Graded Materials belong to a class of advanced materials characterized by gradual variation in composition and structure over their changing volume, resulting in corresponding changes in the material properties. These graded materials could also be chosen to harness its shape changing properties, designed and engineered for a specific set of functions and applications. Within FGMs, the different micro-structural phases have different functions. FGMs attain their multifunctional from the smooth transitions of constituent phases. They can be designed and engineered for a specific set of functions and applications, such being responsive towards specific stimuli (as a 4D printed part), or for mechanical shock resistance, thermal insulation, catalytic efficiency and relaxation of thermal stresses (Oxman, 2011; Oxman 2012).

Most conventional AM techniques utilise a single material throughout the production process. This design procedure has been implemented in the optimisation design process of tissue engineering scaffolds. Chua et al. (2008; 2010) and Sudarmadji et al. (2011) presented a methodology for 
designing functionally graded tissue engineering scaffolds. It was composed of a library 13 polyhedral geometric models which would be used with Boolean operations and anatomic models to design an optimum scaffold. Another design concept developed by Yoo (2012a), considered a novel heterogeneous modelling methodology for designing tissue engineering scaffolds, with precisely controlled porosity and internal architectures using triple periodic minimal surfaces according to the scaffold's biological and mechanical requirements. Yoo (2013) presented another general design framework for 3D internal scaffold architectures to match desired mechanical properties and porosity simultaneously, by introducing an implicit interpolation algorithm based on the radial basis functions. Later on, Yoo (2014) developed another design concept of multi-void triple periodic minimal surfacebased scaffolds that increases the surface area to volume ratios of conventional triple periodic minimal surfaces scaffolds. Hazlehurst et al. (2014) considered the flexural characteristics of laser melted cobalt chrome femoral stems, by using a combination of mechanical testing and finite element analysis. A functionally graded design methodology was considered in order to reduce the weight and stiffness of the femoral stems. Three separate functionally graded designs were investigated by incorporating square pore cellular structures of varying density. The results confirmed that selective laser melting can repeatedly manufacture a functionally graded femoral stem that is $48 \%$ lighter and $60 \%$ more flexible than a traditional fully dense stem. In the field of medical applications, the use of functionally graded models has increased exponentially presenting very positive results within several domains (biological, mechanical, etc.). Existing systems such as Fused Deposition Modelling and 3D screen printing have demonstrated the feasibility of multi-material printing. Commercial FDM systems such MakerBot and Ultimaker extrusion machines include dual or even triple extruder heads but the materials cannot be mixed. The work by Riecker et al. (2014) proposed printing with a two material layout to demonstrate multi-material 3D screen printing that is capable of producing complex inner structures, undercuts and complete hollow structures. More recently, Sitthi-Amorn et al. (2015) proposed a novel multi-material 3D printing platform that is low-cost, highresolution and extensible with a material library of 15 different materials.

In contrast with conventional single-material and multi-material AM which focuses on shape-centric prototyping, FGAM focuses on material-centric fabrication by highlighting the structure-property relationship. This process is characterized by simultaneous synthesis and densification of threedimensional objects that are driven by the pre-determined organisation of the deposited material as opposed to only its shape and form. Scholars have used other terms such as Functionally Graded Rapid Prototyping (FGRP) and Varied Property Rapid Prototyping (VPRP) although in principle, they refer to the same process. According to Oxman (2011), FGAM is an integrated design approach and a technological framework to model, analyze and fabricate components that are characterized by gradual variations in composition and structure over volume, corresponding with multiple and continuously varied functional constraints. FGAM parts attain their multi-functional status from their property gradation, which is achieved by mapping performance requirements and allocating the material structures throughout the 3D space (Figure 1).

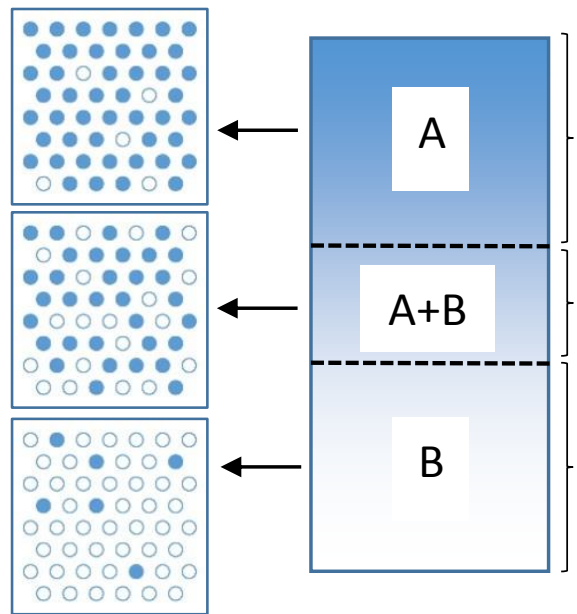

Phase 1 (particles) with phase 2 as matrix

Transition region

Phase 2 (particles) with phase 1 as matrix 
Figure 1: Continuously graded microstructure of FGMs

In order to achieve this functional design, Oxman (2012) proposed a Finite Element Synthesis approach to design physical prototypes with variable material properties by applying the finite element method in the early stages of the design process. This approach assumes and facilitates the distribution of multiple material properties as a function of site-specific constraints from the structural simulations. Brackett et al. (2014) proposed an error diffusion methodology to convert a continuous tone image into binary form, resulting in a functionally graded cellular structure based on a spatial variation of cell size. Effects of two control parameters, greyscale value and resolution on the resulting cell size measures were considered. Results demonstrate that the variation in cell edge length was greatest for the Voronoi connection scheme, particularly at certain parameter combinations. Relationships between these parameters and cell size were identified and applied to an example, where the target was to control the minimum and maximum cell size. The controlled variation of the FGMs within the 3D space can be produced by controlling the ratios in which two or more materials that are mixed prior to the deposition and curing of the substance (Mahamood, 2012). Other researchers including Birman (2007) defined FGAM as the coupling effect of materials through sandwich configurations. Kieback (2003) and Hascoet (2011) both defined FGAM as the process of building objects with compositional and microstructural gradients using AM technology, creating an optimum combination of component properties such as weight, surface hardness, wear resistance, impact resistance and toughness; or to produce material gradients to control physical, chemical, biochemical, mechanical properties or biocompatibility characteristic with a complex morphology. Although the work by Rener et al. (2014) successfully demonstrated the use of two extruders of a low-end Fused Deposition Modelling 3D printer to produce continuous tone imagery by finely interleaving two colours while minimizing the switching between print heads, the blending of colours (similar to ZCorp powder-based, colour 3D printing machines) does not constitute a mixing or blending of material composition and thus is not considered to be within the realm of FGAM. FGAM establishes a radical shift from contour modelling to material-performance modelling. The aim of FGAM is to fabricate performance-based geometrical components driven by their graduated properties and material behaviour to support the integration of functions.

In summary, we clarify that Functionally Graded Additive Manufacturing (FGAM) is a variable property rapid fabrication method (VPM) aimed to dynamically mix and vary the ratios of materials within a three dimensional volume to produce a seamless integration of monolithic functional structures with varied properties using the same machine. The use of FGAM can achieve properties such as varying the level of glass transition, density, elasticity and translucency. The differences in micro-structural phases contribute to different material properties within the singular component. In short, Functionally Graded Additive Manufacturing (FGAM) is defined as a single additive manufacturing process that includes the gradational mixing of materials to fabricate freeform geometries with variable-properties within one component. Figure 2 illustrates the differences between a single-material AM component, a multi-material AM component, and a FGAM component.
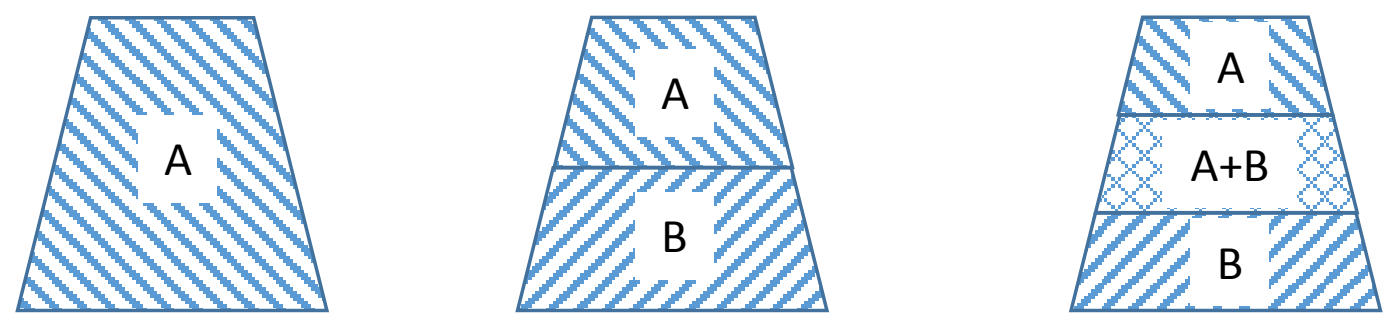
Figure 2: Differences in single-material, multi-material and FGAM prints.

\subsection{Clarifying Functionally Graded Additive Manufacturing and 4D Printing}

One area of research that has gained popularity is the use of smart materials that can sense fluctuations in the external environment and generate a response by either changing the material properties or geometries (Varadan et al., 1992). Smart materials have behaviours such as self-sensing, self-actuating and can change their shape when the external stimulus is applied. This "smart" behaviour is known as the shape memory effect and sources of external stimulus include physical or chemical means such as temperature, humidity, light, acidity level or magnetic sources (Berg et al., 2014). When AM techniques and smart materials are combined, the printed structures no longer become static but are active. This approach is known as 4D Printing, whereby the design and the geometric programme enables the capacity for a change of state such as folding, curling, expanding, contracting and other transformations (Pei, 2014a, Pei, 2014b; Tibbits et al., 2013).

At a commercial level, production of $4 \mathrm{D}$ printed parts can be realised using the Connex range of printers from Stratasys Ltd. that utilise the PolyJet process that deposits multiple materials being controlled through a CAD interface (Vaezi et al., 2013). Although these systems are capable of producing high-resolution components, Polyjet machines and materials are expensive and the hardware and software architectures are proprietary and inextensible (Sitthi-Amorn et al., 2015). Khoo et al. (2015) defined that 4D printing can be achieved through the use of a single smart material or a mixture of smart materials and conventional materials to produce active nanocomposites, shape memory alloys and shape memory polymers (Kim et al. 2014; Raviv et al., 2014; Ge et al., 2014). Zarek et al. (2016) claimed that the growing interest in 4D printing has been kindled by the implications for responsive structures that can be used in soft robotics (McEvoy and Correll, 2015), printable actuators (Bakarich et al., 2015), medical devices (Saatchi et al. 2015, Gao et al. 2016) and relevant towards industries such as textiles ( $\mathrm{Hu}$ et al., 2012), defence (Felton et al. 2014) and aerospace (Leng et al., 2011). Zarek et al. (ibid) proposed a method using Methacrylated semicrystalline polymer to fabricate objects that exhibit thermally triggered shape memory behaviour. They demonstrated the use of the polymer to produce dynamic, shape-changing jewellery and a shoe accessory using the method of Digital Light Processing. By accurately specifying material properties in different sections, they demonstrated that the deformed SMP component can successfully return back to the original configuration. Yu et al. (2015) described Functionally Graded SMPs with sequential shape recovery properties that can be achieved by post curing SMPs on a surface with a linear temperature gradient, using different sections of the material with increasing glass transition temperatures $(\mathrm{Tg})$ that consequently react differently. Other scholars also suggested that SMPs can be produced using UV polymerization with patterned photo filters and co-extrusion with specially designed gradient distribution (Mather et al., 2009; Zhu et al., 2006; Wen et al., 2004).

This section has described that while 4D Printing involves the combination of smart materials and Additive Manufacturing to produce shape-changing parts that are responsive to stimuli, Functionally Graded Additive Manufacturing (FGAM) is process of using an Additive Manufacturing machine to dynamically mix and vary the ratios of materials within a three dimensional volume to produce a single component. 4D Printed parts can be made of either single material, multi-material or Functionally-graded material, in which the substances are able to react to changes in the environment to evoke the shape-memory effect. In contrast, FGAM parts do not necessarily need to 
embed a shape-memory effect and they can be static throughout the lifecycle. It is generally acknowledged that the FGAM parts can be characterized by the variation in composition and structure gradually over a defined volume (Chiu, 2008; Yu et al., 2015).

\subsection{The Modelling Process of FGAM Parts}

With respect to current Computer Aided Design (CAD), existing limitations lie in employing digital entities (programming, designing and simulation) that can accurately describe the micro-scale properties of materials and to generate the toolpaths for fabrication. Current CAD tools employed for AM technologies have remained fairly traditional and no material information is taken into consideration in this intermediate file format. The typical input data format from a CAD system is usually a triangular facet model such as a *.STL file in which only a homogeneous object can be represented (Chiu, 2008). The paper by Oxman (2011) recognized that one of the greatest gaps in FGAM where mainstream CAD applications still do not support the descriptions of materials within the artefact. Although emerging approaches such as the use of voxels (voxel-based graphics methodologies), finite-elements (FEM/ FEA), particle system elements and vague discrete modelling elements (VDM) exist for lattice generation (Aremu, 2016), editing of such formats is difficult by the lack of a robust method to combine and integrate modelling, analysis and the production flow. The major disadvantage of voxel-based systems is the need for additional computational power and long processing times to generate individual voxels and sheets of pixels for every layer. Each voxel must be modified if the design changes (Knoppers, 2004). Furthermore, to convert a voxel model into a common geometric form for AM, the number of facets required to define the lattice as an acceptable file format (such as STL) makes this computationally complex (Aremu, 2016).

To achieve an effective print, the production requirements must be contained in the manufacturing file. Most efforts for AM file standards still appear to be focused on standardising the definition of CAD geometry such as improving the *.STL file through AMF and 3MF file format initiatives (ISO TC261 WG4, 2015; 3MF Consortium, 2015). In addition to the CAD geometry, other important build information and data requirements that are needed include object orientation, support structures, slice structures, machine paths, object packing information, and tolerance data (Pratt et al., 2002). For example, information such as geometry data is independent of manufacturing processes, while other information such as tool paths are dependent on the manufacturing process (Lipman and McFarlane, 2015). The most prominent effort to date is the AMF format which is recognised as an official ISO standard for AM. An upcoming alternative is the 3MF standard that arose as an effort to bridge the gap between hardware and software systems. Both are based on the extensible markup language $(\mathrm{XML})$, in which they follow a standardised, text-based, human readable encoding format following the open XML specification (Bray et al., 2008). Both AMF and 3MF are open and evolving standards that are intended to handle the large amounts of design, geometric and material data required by AM. However, both formats are still unpopular and industry take-up has been low. The de facto format being used for AM today is still the *.STL file which is incapable of incorporating material and manufacturing information.

For FGAM to take off, it requires a new approach of Computer-Aided Engineering (CAE) analysis that can specify, model and manage material information for Local Composition Control (LCC) (Chiu, 2008). The LCC data can be sent to the machine in a layer-by-layer pixel sheet so that when they are stacked, they are represented as voxel clouds. Advanced data driven additive fabrication technologies permit the ability to strategically control the density and directionality of material substance to generate the overall form. The software is required to manage the layering or compounding of dissimilar materials, such as to regulate the variation of stiffness by graduating the distribution of hard and soft materials throughout the geometry. Vidimce et al. (2013) proposed a programmable pipeline and architecture 
for direct specification of multi-material objects that outputs multi-material rasters for each layer. Taking a step further, Vidimce et al. (2016) proposed a Hierarchical Material Design for MultiMaterial Fabrication through an interactive and visual process for designing spatially-varying material properties such as appearance, mechanical and other optical aspects. FGAM shares a nearly identical process modelling procedure as the conventional AM process where parts are produced directly from digital data. However, Muller $(2012,2014)$ suggested that the key difference in FGAM is that it introduces a feature of toolpath planning which influences the material distribution of the manufactured parts and the process modelling of FGAM is summarized in Table 1.

\begin{tabular}{|l|l|}
\hline $\begin{array}{l}\text { Step 1: } \\
\text { Description of the } \\
\text { Part Geometry and } \\
\text { Material Distribution }\end{array}$ & $\begin{array}{l}\text { Description of the geometry and the material distribution, followed by } \\
\text { classifying the material distribution (the dimension of the gradient). Three } \\
\text { criterions are required: the dimension of the gradient, the shape of } \\
\text { composition surfaces and the distribution of composition surfaces. }\end{array}$ \\
\hline $\begin{array}{l}\text { Step 2: } \\
\text { Determination of } \\
\text { manufacturing } \\
\text { strategies }\end{array}$ & $\begin{array}{l}\text { Material data that concerns the chemical composition and characteristics } \\
\text { of the two (or more) materials used is gathered. The material distribution } \\
\text { and orientation of slices are defined. The toolpaths are evaluated and } \\
\text { calculated. The mathematical data is used to find the most appropriate } \\
\text { manufacturing strategy. }\end{array}$ \\
\hline $\begin{array}{l}\text { Step 3: } \\
\text { Numerical Control }\end{array}$ & $\begin{array}{l}\text { Numerical Control (NC) programming, involving paths and process } \\
\text { parameters is generated with the G programming language (ISO 6983) } \\
\text { from the toolpath route. A 3D grid with machine data and the material } \\
\text { distribution is generated to the defined paths. }\end{array}$ \\
\hline $\begin{array}{l}\text { Step 4: } \\
\text { Manufacturing }\end{array}$ & $\begin{array}{l}\text { The NC program is used by a CNC controller. The operation involves } \\
\text { fabricating slices in order to build three dimensional cross-section profiles } \\
\text { to construct the component layer by layer with pre-determined specific } \\
\text { material deposition. The file is sent to the AM machine for the production } \\
\text { sequence to commence. }\end{array}$ \\
\hline
\end{tabular}

Table 1: The Modelling Process of FGAM Parts

\subsection{Key Benefits and Limitations of FGAM}

FGAM provides a new manufacturing route that can expand the potential of prototyping morphologically complex and customized parts, overcoming various limitations in processing materials by conventional methods (Khoo, 2015). The methodology of FGAM can also be regarded as a means of achieving 4D printing where the use of two or more stimuli-responsive materials; or stimuli-responsive materials with conventional materials; are mixed and printed, and the final part is capable of reacting to the environment. FGAM presents a high level of seamless material integration, eliminating sharp interfaces where two materials meet at locations that component failure may occur. By introducing a functionally graded interlayer, the piezoelectric effect could be used to suppress the dynamic or static response, while grading may result in reduced stresses and deformations (Birman, 2007). FGAM can produce highly customizable features with integrated functionalities to consider load-bearing and structural strain. The approach of FGAM enhances component optimization of material properties relative to their structural performance, thereby potentially reducing in-plane and transverse stresses, improving residual stress distribution, enhancing thermal management, achieving higher fracture toughness, and lowering the stress intensity (Birman, 2007). According to Oxman (2011), dynamic gradation and the application of homogeneous materials within a single AM part offers improvements in structural and environmental performance, enhances material efficiency by reducing weight, promoting material economy by reducing material processing, as well as decreasing the energy input in the product's generation and lifespan. The amount of support 
materials may also potentially decline in cases where minimum support is needed due to geometrical simplicity. The ability to 3D print with variable properties can potentially eliminate the need for support material by varying the relative thickness and structural strength of components in regions of under cuts and areas that require more strength to self-stabilize in the build process and also when the part begins to dry out. In addition, FGAM-driven processes offer variable property support where parts could be removed with ease as stronger support pieces are able to cling onto the fragile areas without breaking.

Chiu (2008) highlighted the main obstacles for FGAM whereby AM production of heterogeneous compositions can only be fulfilled by utilizing multiple printer heads to deposit different materials and / or binders on different parts of the layers of the models during the single printing process. The fabrication process of current FDM technologies are still largely limited to using one material per print. Currently, single-head nozzles can only assign material homogeneously and material graduation is usually achieved by manual means of fabricating multiple parts and then assembling them in a post-print process. FGAM parts are also prone to high internal defects and it will be a challenge for the FGAM system to accurately determine the distribution of constituent phases and properties throughout the component. There is a need to investigate the pre- and post-print procedures to guarantee a higher level of reliability, and to also achieve a predictable distribution of material constituents and properties throughout the structure (Birman, 2007). Current AM technologies in terms of hardware and software are still not set up to represent graduation and variation of properties within solids such as varied densities in concrete, varied elasticities in rubber, or varied translucencies in glass. The design process is constrained to the assignment of discrete (individually separated/ distinct) and homogeneous (single) material properties to a given shape (Oxman, 2011). Present FGAM processes are only capable of fabricating non-functional test-pieces and small components with simple morphology and discrete multi-material distribution (Muller, 2014). To move to functional parts, Muller (2012) further emphasized the need for greater control of all build parameters with an optimal manufacturing strategy; as well as the need for tightly-integrated Computer-Aided Engineering software as described in the earlier sections.

In order to overcome the current limitations, Royo et al. (2015) presented a seamless computational workflow for the design and direct digital fabrication of multi-material and multi-scale structured objects. The workflow encodes for and integrates domain-specific meta-data relating to local, regional and global feature resolution of heterogeneous material organizations. In their work, they focused on water-based materials and demonstrated their approach by additively manufacturing diverse constructs associating shape-informing variable flow rates and material properties to meshfree geometric primitives. The proposed workflow enables virtual-to-physical control of constructs where structural, mechanical and optical gradients are achieved through a seamless design-tofabrication tool with localized control. An enabling technology combining a robotic arm and a multisyringe multi nozzle deposition system was presented.

\subsection{Conclusion}

Oxman (2011) described that existing AM processes only serve as a prototyping method with limited functionality and printed parts are mainly used for visual representations. The basic strategy of current conventional AM technologies is to assign material properties to pre-shaped components, focusing around the geometrical description of form as a property-less feature. They do not take graded properties into account and the current technology's set up is limited in capacity and scale. Although there is a growing trend of using AM for end-use parts for aerospace and medical applications, for AM to enter new markets, the use of FGAM enables a more effective approach of allocating materials according to the required performance. This paper defines Functionally Graded 
Additive Manufacturing (FGAM) as a single AM process that includes the gradationally mixing of materials to fabricate freeform geometries with variable-properties within one component. We also clarify that 4D Printing is a fabrication technique in which Additive Manufacturing and smart materials are used to produce geometrically designed parts that have the ability to change when exposed to an environmental stimuli (Pei 2014a; Pei 2014b; Tibbits et al., 2013).

FGAM requires better computational tools for modelling, simulation and fabrication as current CAD systems are incapable of supporting the FGAM workflow. Certainly, other factors such as strength, type of materials, etc. must be taken into account when selecting an appropriate process for FGAM (Chiu, 2008). This is in line with Mahamood (2012) who highlighted that more research needs to be conducted on improving the performance of FGAM processes through extensive characterization of functionally graded materials (FGMs) to generate a comprehensive database and to develop a predictive model for proper process control. It is expected that future work will focus on both material characterization as well as seamless FGAM control processes.

\subsection{References}

1. 3MF Consortium (2015). 3D Manufacturing Format - Core Specification \& Reference Guide v1.1. Available at: http://3mf.io/wp-content/uploads/2016/03/3MFcoreSpec_1.1.pdf (Accessed: 20 December 2016).

2. Aremu, A.O., Brennan-Craddock, J.P.J., Panesar, A., Ashcroft, I.A., Hague, R.J.M., Wildman R.D. and Tuck, C. (2016). A Voxel-based Method of Constructing and Skinning Conformal and Functionally Graded Lattice Structures Suitable for Additive Manufacturing. Additive Manufacturing (13). pp. 1-13.

3. Bakarich, S.E., Gorkin, R., Panhuis, M.i.h. and Spinks, G.M. (2015). 4D Printing with Mechanically Robust, Thermally Actuating Hydrogels. Macromolecular Rapid Communications (36). pp. 12111217.

4. Berg, G.J., McBride, M.K., Wang, C. and Bowman, C.N. (2014). New Directions in the Chemistry of Shape Memory Polymers. Polymer, 55(23). pp. 5849-5872.

5. Birman, V. and Byrd L.W. (2007). Modelling and Analysis of Functionally Graded Materials and Structures. Applied Mechanics Reviews 60(5). pp. 195-216.

6. Brackett, D.J., Ashcroft, I.A., Wildman, R.D. and Hague, R.J.M. (2014) "An error diffusion based method to generate functionally graded celular structures", Computers and Structures, 138:102111.

7. Bray, T., Paoli, J., Sperberg-McQueen, C.M., Maler, E. and Françoise, Y. (2008). Extensible Markup Language (XML) 1.0 (Fifth Edition). W3C. Available at: https://www.w3.org/TR/REC-xml (Accessed: 7 December 2016).

8. Chiu, W.K. and Yu, K.M (2008). Direct Digital Manufacturing of Three-dimensional Functionally Graded Material Objects. Computer-Aided Design 40(12). pp. 1080-1093.

9. Chua, C.K., Sudarmadji, N. and Leong, K.F. (2008) "Functionally graded scaffolds: the challenges in design and fabrication processes", in Virtual and Rapid Manufacturing: Advanced Research in Virtual and Rapid Prototyping, Edited by P.J. Bártolo et al, CRC Press, 115-120.

10. Chua, C.K., Sudarmadji, N., Leong, K.F., Chou, S.M., Lim, S.C. and Firdaus, W.M. (2010) "Process flow for designing functionally graded tissue engineering scaffolds", in Innovative Developments in Design and Manufacturing, Edited by P.J. Bártolo et al, CRC Press, 45-49.

11. Felton, S., Tolley, M., Demaine, E., Rus, D. and Wood, R. (2014). A Method for Building SelfFolding Machines. Science. 345(6197). pp. 644-646.

12. Gao B., Yang, Q., Zhao, X., Jin, G., Ma, Y. and Xu, F. (2016). 4D Bioprinting for Biomedical Applications. Trends in Biotechnology 34(9). pp. 746-756. 
13. Ge, Q., Qi, H.J., and Dunn, M.L. (2013). Active Materials by Four Dimension Printing. Applied Physics Letters (103) 131901-1-5.

14. Hascoet, J.Y., Muller, P. and Mognol, P. (2011). Manufacturing of Complex Parts with Continuous Functionally Graded Materials (FGM). Institut de Recherche en Communications et Cybernetique de Nantes (UMR CNRS 6597).

15. Hazlehurst K.B., Wang, C.J. and Stanford, M. (2014) "An investigation into the flexural characteristics of functionally graded cobalt chrome femoral stems manufactured using selective laser melting",Materials and Design, 60:177-183.

16. Hu, J., Zhu, Y., Huang, H. and Lu, J. (2012). Recent Advances in Shape-Memory Polymers: Structure, Mechanism, Functionality, Modeling and Applications. Progress in Polymer Science. 37(12). pp. 1720-1763.

17. ISO TC261 WG4 (2015). ISO/ASTM 52915:2014 Specification for Additive Manufacturing File Format (AMF) Version 1.2. Geneva, Switzerland: ISO/ASTM International.

18. Khoo, Z.X., Teoh, E.M.J., Liu, Y., Chua, C.K., Yang, S., An, J., Leong, K.F. and Yeong, W.Y. (2015). 3D Printing of Smart Materials: A Review on Recent Progresses in 4D printing. Virtual and Physical Prototyping. 10(3). pp. 103-122.

19. Kieback, B., Neubrand, A. and Riedel, H. (2003). Processing Techniques for Functionally Graded Materials. Materials Science and Engineering. Papers from the German Priority Programme (Functionally Graded Materials). 362(1-2). pp. 81-106.

20. Kim, K., Zhu, W., Qu, X., Aaronson, C., McCall, W.R., Chen, S. and Sirbuly, D.J. (2014). 3D Optical Printing of Piezoelectric Nanoparticle-Polymer Composite Materials. American Chemical Society Nano. 8(10). pp. 9799-9806.

21. Knoppers, G.E., Gunnink, J.W., van den Hout, J. and van Vliet, W.P. (2004). The Reality of Functionally Graded Material Products. Industrial Prototyping, TNO Industrial Technology, De Rondom 1, 5600 HE Eindhoven, The Netherlands.

22. Leng, J., Lan, X., Liu, Y. and Du, S. (2011). Shape-Memory Polymers and their Composites: Stimulus Methods and Applications. Progress in Materials Science. 56(7). pp. 1077-1135.

23. Lipman, R.R. and McFarlane, J.S. (2015). Exploring Model-Based Engineering Concepts for Additive Manufacturing. In: Proceedings of the 26th Solid Freeform Fabrication Symposium. 26th Solid Freeform Fabrication Symposium, Austin, TX, USA. Available at: http://www.researchgate.net/publication/281285803_Exploring_Model- Based_Engineering_ Concepts_for_Additive_Manufacturing (Accessed: 18 December 2016).

24. Mahamood, R.M., Akinlabi, E.T., Shukla, M. and Pityana, S. (2012). Functionally Graded Material: An Overview. Proceedings of the World Congress on Engineering 2012 Vol III WCE 2012, July 4-6, 2012, London, UK.

25. Mather, P.T., Luo, X.F. and Rousseau, I.A. (2009). Shape Memory Polymer Research. Annual Review of Materials Research (39). pp. 445-471.

26. McEvoy, M. and Correll, N. (2015). Materials that Couple Sensing, Actuation, Computation, and Communication. Science 347(6228). 1261689-1-1261689-8.

27. Muller, P., Hascoet, J.Y. and Mognol, P. (2012). Functionally Graded Material (FGM) Parts: From Design to the Manufacturing Simulation. Proceedings of the ASME 2012 11th Biennial Conference on Engineering Systems Design and Analysis ESDA2012 July 2-4, 2012, Nantes, France.

28. Muller, P., Hascoet, J.Y. and Mognol, P. (2014). Toolpaths for Additive Manufacturing of Functionally Graded Materials (FGM) Parts. Rapid Prototyping Journal. 20(6). pp. 511-522.

29. Oxman, N (2011). Variable Property Rapid Prototyping. Virtual and Physical Prototyping 6(1). pp. 3-31.

30. Oxman, N. (2012). Finite Element Synthesis. Proceedings of VRAP: Advanced Research in Virtual and Rapid Prototyping. In: "Innovative Developments in Virtual and Physical Prototyping ", P.J. Bártolo et al. (eds.), Taylor \& Francis. 
31. Oxman, N., Keating, S. and Tsai, E. (2011). Functionally Graded Rapid Prototyping. Proceedings of VRAP: Advanced Research in Virtual and Rapid Prototyping. In: "Innovative Developments in Virtual and Physical Prototyping ", P.J. Bártolo et al. (eds.), Taylor \& Francis.

32. Pei, E. (2014a). 4D Printing - Revolution or Fad? Assembly Automation. 34(2). pp. 123-127.

33. Pei, E. (2014b). 4D Printing: Dawn of an Emerging Technology Cycle. Assembly Automation. 34(4). pp. 310-314.

34. Pratt, M.J., Bhatt, A.D., Dutta, D., Lyons, K.W., Patil, L. and Sriram, R.D. (2002). Progress Towards an International Standard for Data Transfer in Rapid Prototyping and Layered Manufacturing, Computer-Aided Design. 34(14). pp. 1111-1121. Available at: http://www.sciencedirect.com/science/article/pii/S0010448501001890 (Accessed: 18 November 2016).

35. Raviv, D., Zhao, W., McKnelly, C., Papadopoulou, A., Kadambi, A., Shi, B., Hirsch, S., Dikovsky, D., Zyracki, M., Olguin, C., Raskar, R. and Tibbits, S. (2014). Active Printed Materials for Complex SelfEvolving Deformations. Scientific Reports. 4 (7422).

36. Reiner, T., Carr, N., Měch, R., Št'ava, O., Dachsbacher, C. and Miller, G. (2014). Dual-color Mixing for Fused Deposition Modeling Printers. Computer Graphics Forum. 33(2). pp. 479-486.

37. Riecker, S., Studnitzky, T., Andersen, O. and Kieback, B. (2014). 3D Multi-Material Metal Printing of Delicate Structures. Euro PM2014 - AM: Technologies. Powder Metallurgy European Congress \& Exhibition. 21-24 September 2014. Salzburg, Austria.

38. Royo, J.D., Soldevila, L.M. and Oxman, N. (2015) "Flow-based fabrication: An integrated computational workflow for design and digital additive manufacturing of multifunctional heterogeneously structured objects", Computer-Aided Design, 69:143-154.

39. Saatchi, M., Behl, M., Nöchel, U. and Lendlein, A. (2015). Copolymer Networks From Oligo ( $\varepsilon$ caprolactone) and n-Butyl Acrylate Enable a Reversible Bidirectional Shape-Memory Effect at Human Body Temperature. Macromolecular Rapid Communications. (36). pp. 880-884.

40. Sitthi-Amorn, P., Ramos, J.E., Wang, Y., Kwan, J., Lan, J., Wang, W. and Matusik, W. (2015) MultiFab: A Machine Vision Assisted Platform for Multi-Material 3D Printing. ACM Transactions on Graphics (TOG). 34(4). Proceedings of ACM SIGGRAPH 2015.

41. Sudarmadji, N., Tan, J.Y., Leong, K.F., Chua, C.K. and Loh, Y.T. (2011) "Investigation of the mechanical properties and porosity relationships in selective laser-sintered polyhedral for functionally graded scaffolds", Acta Biomaterialia, 7:530-537.

42. Tibbits, S., Linor, S., Dikovsky, D. and Hirsch, S. (2013). 4D Printing: Multi-Material Shape Change. Architectural Design. (84). pp. 116-121.

43. Vaezi, M., Chianrabutra, S., Mellor, B. and Yang, S. (2013). Multiple Material Additive Manufacturing - Part 1: A Review. Virtual and Physical Prototyping (8). pp. 19-50.

44. Varadan, V.V., Chin, L.C., and Varadan, V.K. (1992). Modelling Integrated Sensor/Actuator Functions in Realistic Environments. In: First European Conference on Smart Structures and Materials. Forte Crest Hotel, Glasgow, UK.

45. Vidimče, K., Kaspar, A., Wang, Y. and Matusik, W. (2016). Foundry: Hierarchical Material Design for Multi-Material Fabrication. Proceedings of the 29th Annual Symposium on User Interface Software and Technology. October 16-19, 2016, Tokyo, Japan.

46. Vidimče, K., Wang, S.P., Ragan-Kelley, J. and Matusik, W. (2013). OpenFab: a Programmable Pipeline for Multi-Material Fabrication. ACM Transactions on Graphics (TOG). 32(4).

47. Wen, B.Y., G. Wu, and J. Yu (2004). A Flat Polymeric Gradient Material: Preparation, Structure and Property. Polymer. 45(10). pp. 3359-3365.

48. Yoo, D.J. (2012a) "Heterogeneous porous scaffold design for tissue engineering using triply periodic minimal surfaces", Precision Engineering and Manufacturing, 13(4):527-537. 
49. Yoo, D.J. (2013) "Heterogeneous Porous Scaffold Design Using the Continuous Transformations of Triply Periodic Minimal Surface Models", International Journal of Precision Engineering and Manufacturing, 14(10):1743-1753.

50. Yoo, D.J. (2014) “Advanced Porous Scaffold Design using Multi-Void Triply Periodic Minimal Surface Models with High Surface Area to Volume Ratios", International Journal of Precision Engineering and Manufacturing, 15(8): 1657-1666.

51. Yu, K., Ritchie, A., Mao, Y., Dunn, M.L. and Qi, H.J. (2015). Controlled Sequential Shape Changing Components by 3D Printing of Shape Memory Polymer Multi Materials. Procedia IUTAM. (12). pp. 193-203.

52. Zarek, M., Layani, M., Eliazar, S., Mansour, N., Cooperstein, I., Shukrun, E., Szlar, A., Cohn D. and Magdassi, S. (2016). 4D Printing Shape Memory Polymers for Dynamic Jewellery and Fashionwear. Virtual and Physical Prototyping. 11(4). pp. 263-270.

53. Zhu, Y.B., Ning, N.Y., Sun, Y., Zhang, Q. and Fu Q. (2006). A New Technique for Preparing a Filled Type of Polymeric Gradient Material. Macromolecular Materials and Engineering. 291(11). pp. 1388-1396. 